\title{
Editorial
}

\section{MEDICAL INFORMATIC, MI}

\author{
Salah Mostafa \\ Professor of Preventive Medicine and Epidemiology \\ Faculty of Postgraduate Childhood Studies \\ Ain-Shams University \\ * Fellow of American College of Epidemiology
}

A number of different transformation processes can be used to transform data into information. Transformation processes are also known as "data processes "

Some examples of transformation processes include the following:

1- Classification: This involves placing data into categories, e.g. categorizing a variable as gender and age.

2- Rearranging/sorting: This involves organizing data so that items are grouped together or placed into a particular order, e.g. medical patients.

3- Aggregating: This involves summarizing data, e.g. by calculating averages age.

4- Performing calculations, e.g. age among different groups of patients.

5- Selection: This involves choosing or discarding items of data based on a set of selection criteria, e.g. hypertensive patients with coronary heart diseases 
Informatics Triangle, By Salah Mostafa, MD [EGYPT] FACE [USA] Research, Administration, Quality, RAQ; Partnership, Education, and Training, PET

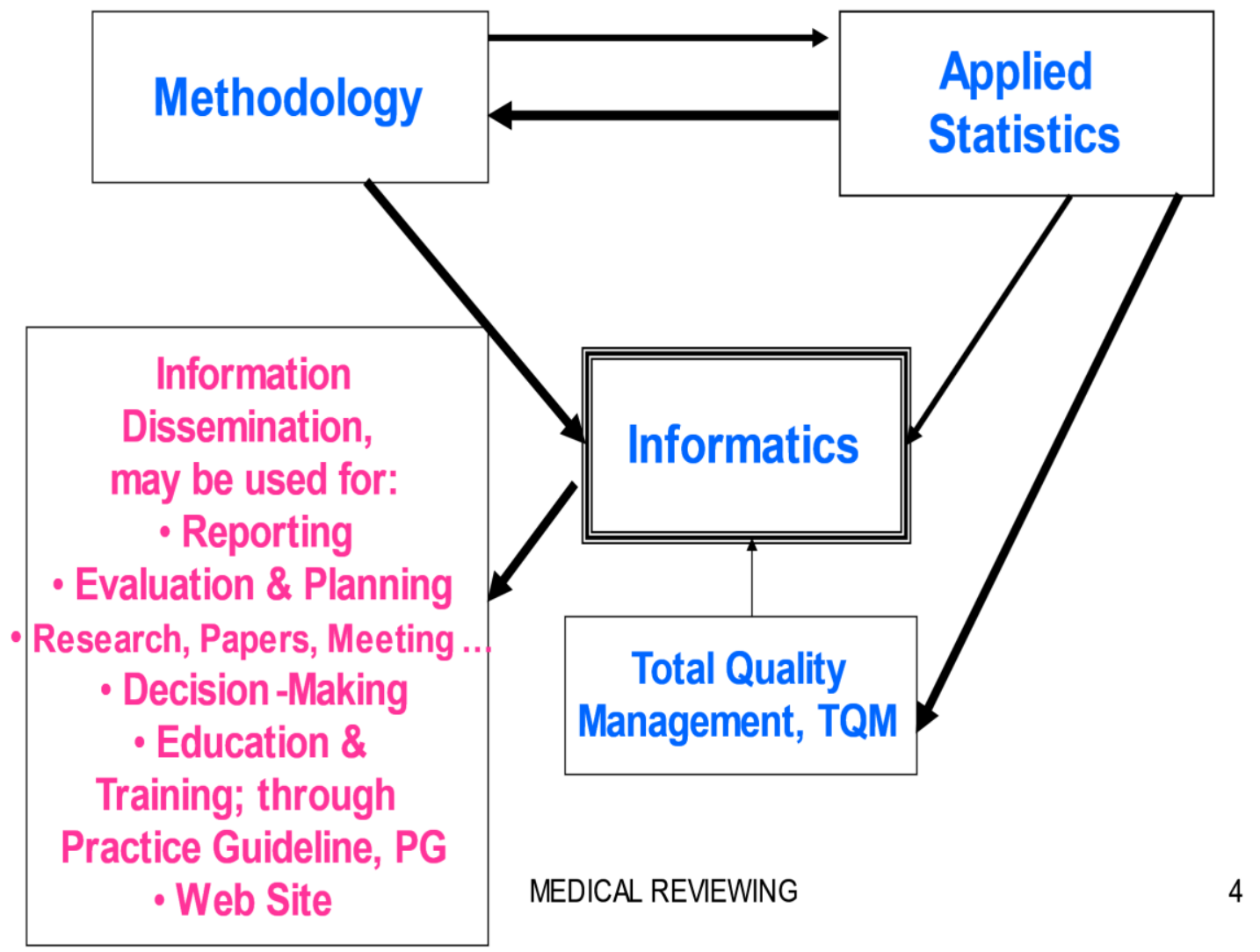

Nearly, most of the activities can be done electronically. Organized medical informatic has been based upon manual methods of recording data. They have been incomplete, costly, burdensome and slow. Mls led us to consider the use of computer-based automation to support, supplement or replace all manual methods; MI will reduce the cost and improve data management.

The $\mathrm{Ml}$ is defined as an organized grouping of personals, equipment, materials and procedures ordered for the accomplishment of unified and applied purpose[s] or objective[s]. On the other hand, Ml are such systems which involve informational Input Form[s], Processing, and Output Form[s], whether these forms take the ways of computer processing, manual, and/or mechanical other forms of processed data. It could be defined also as a set of procedures that collect, store, process, store, and disseminate information for periodic reporting, to decision-making groups, education, and/or training. Availability of capable communications network between medical providers is essential to the success of the mechanisms of information flow. 
Medical practitioners can access patients data in seconds. It provides more legible and better organized report, improves physician ability to predict clinical outcomes, ables to abstract, summarizes and MI lights the important elements of medical files, offers opportunity for integrated data base, and allows updating and inquiry with the use of remote terminals. Aggregated information of many patients will be useful in clinical research.

MI requires a large initial investment than its paper counterpart because of hardware, software and training costs. Enough time must be spent for learning system and training the staff. Computer based systems have the potential for catastrophic failure, thus alternative manual should be available.

The major objective is to combine the functions of data acquisition and storage, information processing and display to create a system that effectively meets information needs. It is to identify and ensure collection of uniformly defined information necessary to plan, manage, operate and evaluate medical system. Also, ensure appropriate access to consistent information and integrate information across service delivery, functional, geographic and jurisdictional boundaries as required. The new system has to minimize capital and operational system cost and continuously evaluate information system in relation to benefits produced and ensure compliance with freedom of information, protection of privacy and documents and files management legislation. However, these goals cannot be achieved without the existence of and compliance with a common set of policies and standards for medical information management.

Functions of MI include manage information that medical professionals need to perform their jobs effectively and efficiently and improve quality and outcome of patient care. Also, it is to increase productivity of the medical unit and assist the doctors to improve their performance. MI will allow to abstract, summarize and light the important elements of medical files, offer opportunity for integrated data base, and allow updating and inquiry with the use of remote terminals. Aggregated information of many patients will be useful in periodic reporting and clinical research.

MI can possess a diverse set of capabilities, usually through the acquisition, of systems, to support departmental activities. Interactions between these systems provide the opportunities to gather further capabilities through the use of databases that collect data from several department systems or that support sharing of events, transactions, and/or link data between systems.

One of the urgent needs is to improve access to medical information. Future Medical care delivery systems will require integrated information management systems to function. It will be impossible to meet the demands for quality assurance, assessment of efficiency and documentation of services without integrated MI. 